\title{
Characteristics of silicon nanocrystals for photovoltaic applications
}

\author{
D. Moore, S. Krishnamurthy, Y.Chao, Q.Wang, D. Brabazonand and P. J. McNally
}

\begin{abstract}
Over the last decade the progress in amorphous and nanocrystalline silicon (nc-Si) for photovoltaic applications received significant interest in science and technology.Advances in the understanding of these novel materials and their properties are growing rapidly. In order to realise nc-Si in the solar cell, a thicker intrinsic layer is required. Due to the indirect band gap in the crystallites, the absorption coefficients of nc-Si are much lower. In this work we have used electrochemical etching techniques to produce silicon nanocrystals of the sizes 3-5 nm. Viable drop cast deposition of Si nanocrystals to increase the thickness without compromising the material properties was investigated by atomic force microscopy, optical microscopy, photoemission spectroscopy and optical absorption methods.
\end{abstract}

Keywords: light absorption, nanocrystalline, photovoltaics, quantum dots

\section{Introduction}

There has been a significant amount of recent research into the possible applications of silicon nanocrystals [1-5]. They provide the possibility of being able to manufacture efficient Si light emitting diodes due to their luminescence properties. However, the possibility of using these silicon nanocrystals in photovoltaic devices has also created enormous interests due to their multiple exciton characteristics. The efficiency can be increased beyond the Shockley-Queisser limit by creating a stack of junctions where the semiconductor comprising each junction has a greater bandgap than the succeeding junction [6]. This could be achieved by varying the size of the silicon nanocrystals to tune the band gap. In this investigation of alkyl-capped nanocrystalline silicon (nc-Si) was prepared and several depositions of nc-Si were onto fused quartz glass substrates were performed using drop cast deposition technique. Surface topography was studied using atomic force microscopy and optical microscopy. Soft X-ray photoemission spectroscopy used to understand the electronic structure of the material. The absorption spectra were measured using an integrating sphere and spectrometer setup. This was performed to better characterise the absorption characteristics of the Si-nc under broadband white light illumination.

Our results suggest that silicon nanocrystals have good potential to replace conventional amorphous Si solar cells through integration into a multi junction stack, for example.

\section{Experimental}

\subsection{Silicon quantum dot preparation}

Lumines-cent silicon nanocrystals were produced by electrochemical etching of silicon. Porous silicon (PS) with a nanoscale internal structure was produced by a standard etching procedure from silicon. The starting point was to remove the native oxide layer from a silicon 
chip by dipping it in hydrofluoric acid (HF) for a short time, washing with de-ionised water and blowing with a stream of nitrogen. The chip was then clamped between two 0-rings in a Teflon cell of diameter 6 or $10 \mathrm{~mm}$, which contains a HF-ethanol (electrolyte) mixture in the ratio 1:1. Due to the hydrophobic character of the clean Si surface, absolute ethanol is usually added to the aqueous solution to increase the wettability of the silicon surface. In fact, ethanoic solutions infiltrate the pores, while purely aqueous HF solutions do not. This is very important for the lateral homogeneity and the uniformity of the PS layer in depth. Another important reason for adding ethanol is that during the reaction hydrogen evolution occurs,bubbles are formed and stick on the Si surface in pure aqueous solutions, whereas they are promptly removed if ethanol is added.

The electrical contact was obtained via thin copper wire in the base of the Teflon cell. A coiled tungsten wire $(0.5 \mathrm{~mm})$ was used as the counter electrode. Silicon nanoparticles were obtained after 15 min etching at current density of $\left(380-400 \mathrm{~mA} / \mathrm{cm}^{2}\right)$. The electrolyte was removed from the cell while the nanocrystals remained on the chip. These particles were dried in a Schlenk flask under vacuum. The dried nanocrystals were introduced into a flask containing a mixture of the $2 \mathrm{~mL}$ of toluene or mesitylene and $0.1 \mathrm{~mL}$ of undecenol solution. The solution was refluxed for 2-6 h under nitrogen atmosphere. During this refluxing process, the whole internal structure of the nanocrystals is modified. This process is termed alkylation. During this reflux only the smallest silicon nanoparticles broke off the PS matrices and dissolved in the solution. The particles have been called silicon nanocrystals due to their size (3-5 $\mathrm{nm}$ in diameter).

2.2 Substrate preparation and deposition For optical absorption characterisation fused quartz glass was used as a substrate. This $1 \mathrm{~mm}$ thick glass was laser cut into $10 \mathrm{~mm} \times 10$ mm square sections and cleaned using Piranha solution (3:1 mixture, concentrated $\mathrm{H}_{2} \mathrm{SO}_{4}$, $30 \% \mathrm{H}_{2} \mathrm{O}_{2}$ ) at $90{ }^{\circ} \mathrm{C}$ for $25 \mathrm{~min}$. The nc-Si was dispersed in dicholoro-methane [7, 8] and then deposited using the drop deposition method. $4 \mathrm{~mL}$ of the solution was dropped per deposition, and the substrate allowed dry for $24 \mathrm{~h}$ in a clean environment. Three depositions were performed in total.

2.3 Absorption measurement In order to measure the effect of the deposited nc-Si on the absorption of broadband light, an integrating sphere with spectrometer setup was designed, see Fig. 1. Integrating spheres have the ability to collect diffuse and specular reflections and transmissions from samples when irradiated with light [9].

As these specialised spheres are coated with highly reflective diffuse coatings, it is possible to average any transmission or reflection over the whole sphere. This makes it possible to obtain an accurate average of any transmitted or reflected light, regardless of the directionality of the light rays. The integrating sphere (Labsphere model 4P-GPS-040-SF) used in this setup was $100 \mathrm{~mm}$ in diameter with three $25.4 \mathrm{~mm}$ diameter ports at $0,90^{\circ}$ and north pole locations, and a $38 \mathrm{~mm}$ port at $180^{\circ}$. A baffle was located between the 90 and $180^{\circ}$ ports to reduce measurement error due to light falling directly on the detector from the sample. To further improve the accuracy of the measurement a cosine corrector was utilised, which aids the elimination of any directionality from the measurement. 


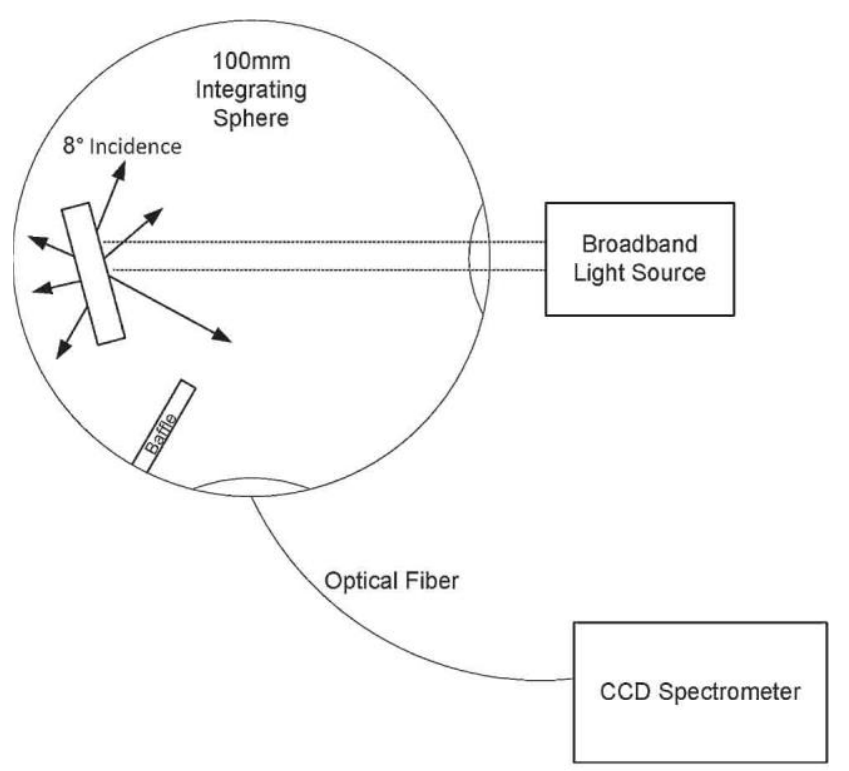

Figure 1 Integrating sphere setup for absorption measurements.

Data below this wave-length are therefore not presented here. The detector integration time for the analysis was $12 \mathrm{~s}$.

To accurately measure the light absorption in the laser processed fused quartz, the samples were held inside the closed sphere with the illumination source incident at $8^{\circ}$ to the normal of the sample. This allowed all the scattered light to be collected including transmitted and reflected, diffuse and specular. By measuring the amount of light collected and subtracting a baseline reference measurement of clear fused quartz with a thin $4 \mathrm{~mL}$ layer of dichloromethane deposited, the absorption could be calculated using Eq. (1).

$$
\text { Absorption }=1-(\mathrm{T}+\mathrm{R})(1)
$$

Where $\mathbf{T}$ is the transmitted light and $\mathbf{R}$ is the reflected light.

\subsection{XPS analysis}

Soft X-ray photoemission was performed at beam 1511, Max-Lab, Lund. The Si 2p photoemission spectrum was taken in normal geometry with $145 \mathrm{eV}$ photons. The total energy resolution is $0.2 \mathrm{eV}$. Binding Energy was referred to the $4 \mathrm{f}$ spectral lines of gold foil, which was in direct contact with the sample, with the binding energy of $84 \mathrm{eV} \mathrm{[10].} \mathrm{For} \mathrm{XPS}$ measurements Si nanocrystals were drop cast onto the graphite or mica surface and rapidly introduced into the vacuum chamber.

\subsection{AFM analysis}

Surface topography analysis of the Si nanocrystals was performed using CII Veeco atomic force microscopy in Tapping Mode. Silicon nanocrystals were drop cast on the surface of the mica/glass substrates and introduced for AFM analysis. Details of the sample preparation for AFM are discussed elsewhere [7]. 


\section{Results and discussion}

Figure 2 shows the optical microscope image of the silicon nanocrystals taken immedi-ately after the third repetition of the drop deposition technique.

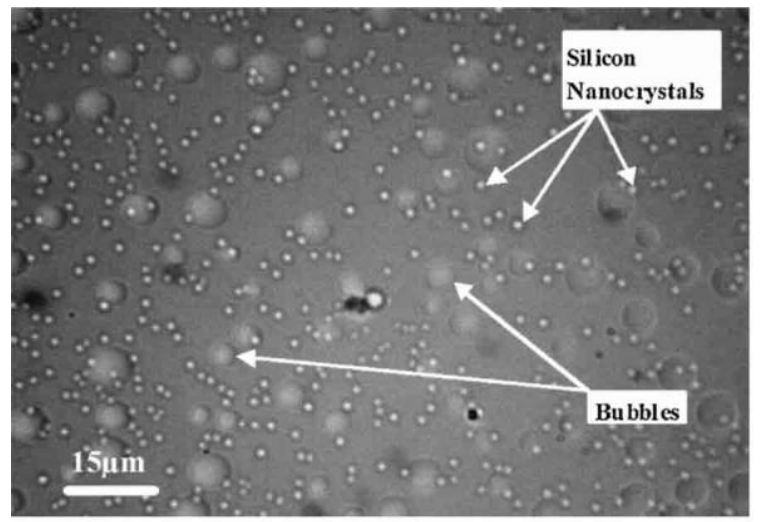

Figure 2 Optical microscope image of nc-Si on substrate shortly after dropdeposition. Bubbles indicate that the solvent is still present.

The silicon nanocrystals appear to be about 1 [Am in diameter, significantly larger than shown by other topographical characterisation methods, such as AFM (Fig. 3). This could be attributed to the formation of clusters of nanocrystals on the surface of the substrate after subsequent depositions [8]. It is clearly visible from this image that the Si nanocrystals do not form a continuous layer on the substrate material. It can also be observed that there are varying degrees of uniformity of Si-nc presented. Some areas are sparsely populated while others are evenly distributed (not shown in the picture).
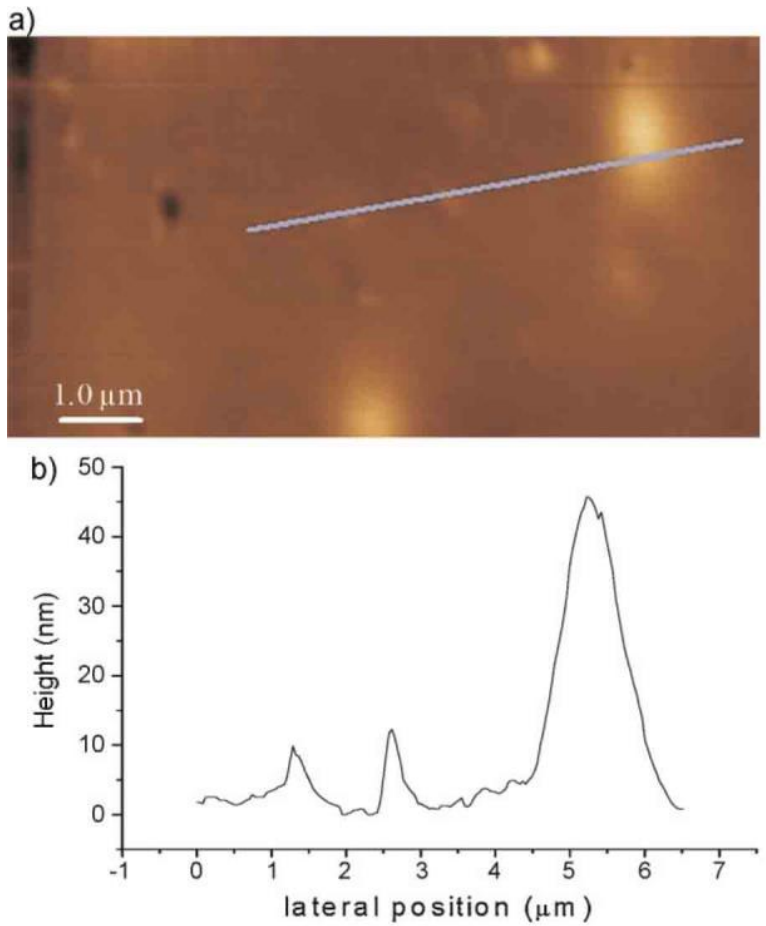

Figure 3 (online colour at: www.pss-a.com) The AFM image was taken on the Si-QDs cast onto mica. From the profile along the line, one can find out the height of clusters was about $10 \mathrm{~nm}$, which is equivalent to the height of two layers of Si-QDs. 
In order to understand the morphology of surface, AFM in tapping mode was performed. Figure 3a represents the double layer deposition of nc-Si on mica/glass. Our previous study on the surface topography by AFM on the similarly produced sample indicated a uniform nanocrystal size distribution of between 3 and $5 \mathrm{~nm}$ [7, 8]. STEM analysis on the same sample has also been previously performed to verify the dimensions of these silicon nanocrystals [8]. Layer-by-layer deposition methods by drop casting the solution on to the surface were attempted. The drop cast deposition technique was used to populate the surface of the mica/glass with two or more layers.

Figure $3 \mathrm{~b}$ shows the cross-sectional analysis of the surface. The size of the nanocrystals is approximately $5 \mathrm{~nm}$ which is consistent with the double layer coating. Clusters of $40 \mathrm{~nm}$ size can also be seen suggesting inhomogeneous evaporation of the solvent.

In order to find the sample composition, element specific X-ray photoemission experiments were carried out. Figure 4 shows the normalised Si $2 p$ spectrum. The spectrum is normalised to incident photon flux. The peak at binding energy $99.5 \mathrm{eV}$ corresponds to the $\mathrm{Si}-\mathrm{Si}$ bonds and the peak at $102 \mathrm{eV}$ corresponds to the silicon_oxygen bonding [7]. In previous work [8] more than two layers were evaporated resulting in a different line shape Si $2 p$ in nc-Si.

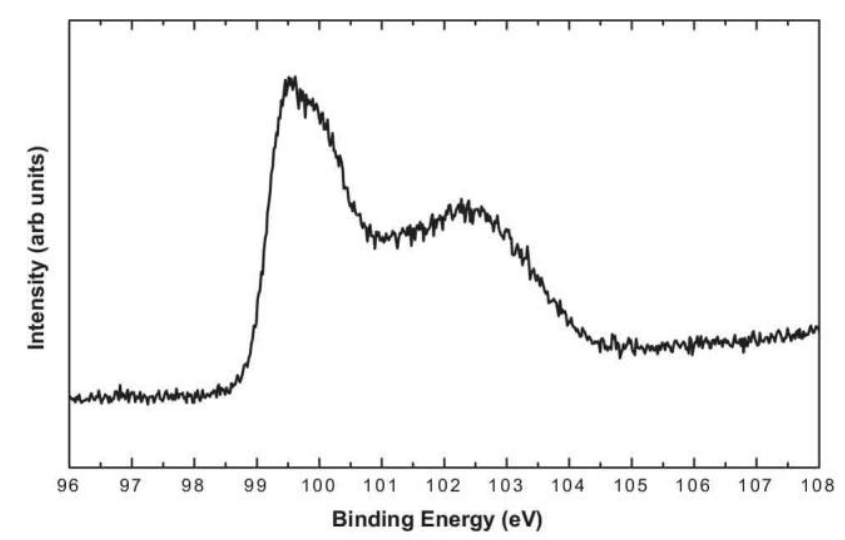

Figure 4 Silicon 2 p photoemission spectra taken at normal geometry with a photon energy of $140 \mathrm{eV}$.

Figure 5 shows the absorption characteristics of the silicon nanocrystals after they were bonded to the glass substrate. The absorption measurement was performed after each repetition of the drop deposition technique and subsequent drying time. It can clearly be seen that there is a small increase in absorption of the incident broadband light by the deposited nanocrystals between 420 and $520 \mathrm{~nm}$. Increases in absorption in the same wavelength range have been found in studies where $\mathrm{Si}$ nanocrystals are embedded in a $\mathrm{SiO}_{2}$ matrix $[11,12]$. Other studies on alkyl-capped Si nanocrystals have found an increase in absorption only from approximately $325 \mathrm{~nm}$ and below [13, 14]. Vasiliev et al. [15] simulated the effect of oxides on the optical absorption of $\mathrm{Si}$ nanocrystals using a confinement model revealing that for certain oxide configurations, the optical absorption gap was further lowered to $2.4 \mathrm{eV}$ (520 $\mathrm{nm})$, which correlates well with our results. 


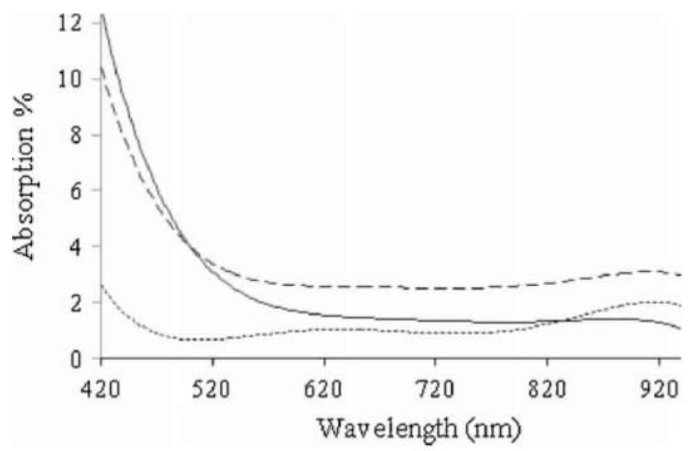

Figure 5 Light absorption characteristics of Si QDs after 1 deposition (dotted line), 2 depositions (dashed line) and 3 depositions (solid line). The absorption of the glass and the solvent have been subtracted from this data.

This indicated that it is the presence of oxides in our nanocrystals, as indicated by the XPS analysis shown in Fig. 4, was causing absorption at lower photon energies. However, it was not possible to achieve a uniform coverage of the substrate with the Si nanocrystals, which may have lead to further inconsistencies in the absorption measurement in this preliminary investigation. It was estimated that in this measurement an experimental error of approximately $1 \%$ existed. The source of this error is due to inhomogeneous distribution of the nanocrystals, and is also due to the variation in the illumination source intensity in the integrating sphere setup. The low population of silicon nanocrystals on the substrate surface led to a lower signal to noise ratio during the measurements. By increasing the number of nanocrystals on the substrate between each measurement an improved signal would be obtained. The variation in light source intensity could be corrected by creating several samples with different nanocrystal popu-lations and performing all of the absorption measurement in one session.

\section{Conclusion}

The absorption of the alkyl-capped $\mathrm{Si}$ quantum dots drop deposited onto a fused quartz substrate was measured using an integrating sphere and spectrometer setup. Subsequent depositions indicated that there is an increase in absorption of broadband light in the 420-500 $\mathrm{nm}$ range. However, the non-uniformity of the drop deposited layer may have lead to inconsistencies in the absorption measurement. Atomic force microscopy confirms the feasibility of double layer depositions and XPS indicates $\mathrm{Si}-\mathrm{Si}$ bonding. Much work needs to be done in order to control the layer-by-layer depositions and to understand the properties of this novel material. By using other deposition techniques, such as evaporation, it will be possible to control the thickness of nanocrystals on the surface of the substrate, which may improve the understanding of the material properties. 


\section{Acknowledgements}

The authors would like to thank the Irish Research Council for Science, Engineering and Technology for funding this research. SK's research was enabled by the Higher Education Authority Program for Research in Third Level Institutions (2007-2011) via the INSPIRE programme.

\section{References}

[1] P. M. Fauchet, Mater. Today 8(1), 26-33 (2005).

[2] J. Valenta, R. Juhasz, and J. Linnros, Appl. Phys. Lett. 80(6), 1070-1072 (2002).

[3] Y. Chen, Y. Ren, R. Xiong, Y. Zhao, and M. Lu, Appl. Surf. Sci. 256(16), 5116-5119 (2010). [4] M. Shiratani, K. Koga, S. Ando, T. Inoue, Y. Watanabe, S. Nunomura, and M. Kondo, Surf. Coat. Technol. 201(9-11), 5468-5471 (2007).

[5] A. J. Nozik and E. Physica, Low-Dimens. Syst. Nanostruct. 14(1-2), 115-120 (2002).

[6] G. Conibeer, M. Green, E. Cho, D. Konig, Y. Cho, T. Fangsuwannarak, G. Scardera, E. Pink, Y. Huang, T. Puzzer, S. Huang, D. Song, C. Flynn, S. Park, X. Hao, and D. Mansfield, Thin Solid Films 516(20), 6748-6756 (2008).

[7] Y. Chao, S. Krishnamurthy, M. Montalti, L. H. Lie, A. Houlton, B. R. Horrocks, L. Kjeldgaard, V. R. Dhanak, M. R. C. Hunt, and L. Siller, J. Appl. Phys. 98,044316 (2005).

[8] Y. Chao, L. Siller, S. Krishnamurthy, P. R. Coxon, U. Bangert, M. Gass, L. Kjeldgaard, S. N. Patole, L. H. Lie, N. O'Farrell, T. A. Alsop, A. Houlton, and B. R. Horrocks, Nature Nanotechnol. 2,486 (2007).

[9] S. L. Storm and A. Springsteen, Spectrosc. Mag. 13(6), 9-14 (1998).

[10] X-ray data booklet, Lawrence Berkeley Laboratory (2009).

[11] X. J. Hao, E. Cho, G. Scardera, E. Bellet-Amalric, D. Bellet, Y. S. Shen, S. Huang, Y. D. Huang, G. Conibeer, and M. A. Green, Thin Solid Films 517(19), 5646-5652 (2009).

[12] P. Mishra and K. P. Jain, Mater. Sci. Eng. B 95(3), 202-213 (2002).

[13] C. Yang, R. A. Bley, S. M. Kauzlarich, H. W. H. Lee, and G. R. Delgado, J. Am. Chem. Soc. 121(22), 5191-5195 (1999).

[14] M. Rosso-Vasic, E. Spruijt, B. V. Lagen, L. D. Cola, and H. Zuilhof, Small 4(10), $1835-$ 1841 (2008).

[15] I. Vasiliev, J. R. Chelikowsky, and R. M. Martin, Phys. Rev. B 65(12), 121302 (2002). 